\title{
GINS2 is a novel prognostic biomarker and promotes tumor progression in early-stage cervical cancer
}

\author{
FEI OUYANG ${ }^{1 *}$, JUNLING LIU ${ }^{2 *}$, MENG XIA $^{1}$, CHUYONG LIN $^{3}$, XIANQIU WU ${ }^{3}$, LIPING YE $^{3}$,

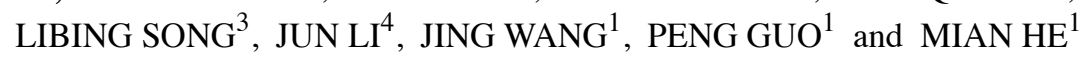 \\ ${ }^{1}$ Department of Gynecology and Obstetrics, The First Affiliated Hospital of Sun Yat-sen University; \\ ${ }^{2}$ State Key Laboratory of Oncology in Southern China, Collaborative Innovation Center for Cancer Medicine, \\ Department of Medical Oncology, Sun Yat-sen University Cancer Center; \\ ${ }^{3}$ State Key Laboratory of Oncology in Southern China, Department of Experimental Research, \\ Collaborative Innovation Center for Cancer Medicine, Sun Yat-sen University Cancer Center; \\ ${ }^{4}$ Department of Biochemistry, Zhongshan School of Medicine, Sun Yat-sen University, Guangzhou, P.R. China
}

Received September 3, 2016; Accepted November 16, 2016

DOI: $10.3892 /$ or.2017.5573

\begin{abstract}
GINS complex subunit 2 (GINS2), a member of the GINS complex, is involved in DNA replication. GINS2 is upregulated in a variety of aggressive tumors. However, its role in cervical cancer carcinogenesis remains to be elucidated. We investigated the clinical significance of GINS2 in patients with early-stage cervical cancer and its biological functions in cervical cancer progression. GINS2 expression was analyzed in cervical cancer cell lines and in 8 matched cervical cancer samples at the mRNA and protein levels using real-time PCR and western blotting, respectively. GINS2 protein expression in 155 paraffin-embedded cervical cancer specimens was validated using immunohistochemistry. Statistical analysis was used to evaluate its clinicopathological significance. Short hairpin RNA interference, anchorage-independent growth ability, colony formation assay, wound healing ability, Transwell assays and western blotting were used to determine
\end{abstract}

Correspondence to: Dr Mian He, Department of Gynecology and Obstetrics, The First Affiliated Hospital of Sun Yat-sen University, 58 Zhongshan Second Road, Guangzhou 510080, P.R. China E-mail: mianhe64@163.com

*Contributed equally

Abbreviations: GINS2, GINS complex subunit 2; OS, overall survival; PLNM, pelvic lymph node metastasis; LNM, lymph node metastasis; MCM, minichromosome maintenance; shRNAs, short hairpin RNAs; GAPDH, glyceraldehyde-3-phosphate dehydrogenase; PBS, phosphate-buffered saline; PVDF, polyvinylidene difluoride; IHC, immunohistochemistry; SCC-Ag, squamous cell carcinoma antigen; HPV, human papilloma virus; ATM, ataxia-telangiectasia mutated; CHK2, checkpoint kinase 2; Rb, retinoblastoma protein; FIGO, International Federation of Obstetrics and Gynecology

Key words: GINS2, cervical cancer, prognosis, proliferation, tumorigenesis, migration, invasion the effects of GINS2 on the aggressive phenotype of cervical cancer cells. There was obvious upregulation of GINS2 in the cervical cancer cell lines and tumor specimens compared to that in the normal cervical tissues. Significant correlations were identified between GINS2 expression and squamous cell carcinoma antigen (SCC-Ag; $\mathrm{P}<0.001)$, deep stromal invasion $(\mathrm{P}=0.021)$, vital status $(\mathrm{P}<0.001)$, recurrence $(\mathrm{P}<0.001)$ and pelvic lymph node metastasis (PLNM; $\mathrm{P}<0.001)$. Moreover, patients with higher GINS2 expression had shorter overall survival (OS) compared to patients with low GINS2 expression. Multivariate analysis revealed that GINS2 may serve as an independent risk factor of poor prognosis in early-stage cervical cancer. In addition, GINS2 downregulation markedly suppressed cell proliferation and tumorigenic ability, as well as cell migration and invasion. Our findings suggest that GINS2 is a novel indicator of PLNM and a valuable prognostic biomarker in early-stage cervical cancer, and subsequently is a valuable molecular target for cervical cancer diagnosis and treatment.

\section{Introduction}

Cervical cancer, a prevalent gynecological malignancy, is the fourth leading cause of cancer-related death in women worldwide, with an estimated 527,600 new cases and 265,700 deaths worldwide in 2012 (1). The majority of cases occur in developing countries and shows a trend for younger patients (2). Despite the decreasing incidence and mortality rate of cervical cancer in recent years owing to improved diagnosis and treatment, the clinical outcome for patients with advanced-stage disease remains bleak (2). Moreover, lymph node metastasis (LNM) can cause higher mortality and recurrence rates, even in patients with early-stage cervical cancer, and definite information on lymph node status is essential for tailoring adjuvant treatment $(3,4)$. However, no sensitive biomarkers specific for indication of LNM, and the early detection and prognosis of cervical cancer are available to date. Therefore, it is urgent to identify novel molecular markers of cervical cancer to 
facilitate a more accurate prediction of clinical outcome and to prescribe effective treatment.

GINS complex subunit 2 (GINS2), also known as PSF2, encodes a protein with a molecular weight of $\sim 21 \mathrm{kDa}$. GINS2 belongs to the GINS complex family that also consists of GINS2, GINS3 and GINS4 (5). The GINS complex has been identified as playing a critical role in the initiation of DNA replication and the cell cycle. Stably interacting with minichromosome maintenance (MCM) 2-7 complex and CDC45, the GINS family functions to correctly establish and maintain DNA replication forks (5). Moreover, GINS components may play a role in cell division, and more accurately, in chromosome segregation (6). GINS2 was reported to be involved in tumorigenesis in several types of cancers. For example, genome-wide gene expression profile analysis revealed that GINS2 is highly expressed in lung carcinoma (7). Zheng et al indicated that GINS2 is correlated with aggressive characteristics of breast cancer, and speculated that it is involved in lung metastasis (8). In addition, enhanced expression of GINS2 was found to promote leukemia cell proliferation and desensitize cells to apoptosis (9). These findings all suggest that GINS2 plays an important role in cancer progression. However, the clinical significance of GINS2 in cervical cancer has not been investigated.

In the present study, we explored the GINS2 expression pattern and its clinical implication and prognostic significance in early-stage cervical cancer. Furthermore, we gained insight into the important functions of this protein in cervical cancer development.

\section{Materials and methods}

Cell lines. Six cervical cancer cell lines, SiHa, HeLa, C33A, Caski, MS751 and ME180, were purchased from the American Type Culture Collection (ATCC; Rockville, MD, USA), and HCC94 and HeLa299, were purchased from the Cell Bank of the Type Culture Collection of the Chinese Academy of Sciences (Shanghai, China). The cell lines were all cultured in RPMI-1640 medium (Gibco, Grand Island, NY, USA) supplemented with $10 \%$ fetal bovine serum (FBS) (HyClone Laboratories, Logan, UT, USA) and $1 \%$ antibiotics.

Patients and samples. In the present study, we enrolled 155 patients with cervical cancer who underwent radical hysterectomy and lymphadenectomy at The First Affiliated Hospital, Sun Yat-sen University. All patients had stage IA2-IIA disease and received treatment from January 2007 to December 2009. The clinical staging and clinicopathological classifications were determined according to the International Federation of Obstetrics and Gynecology (FIGO), 2009. The clinicopathological characteristics of the enrolled cases are summarized in Table I. The follow-up duration for all patients was $>5$ years and the last follow-up date was January 2014. Survival was counted from the date of surgery to the date of death or the last follow-up. Eight paired fresh cervical tumor tissues and the adjacent normal tissues were collected for real-time PCR and western blotting. All paraffin-embedded and fresh tissues used in the present study were obtained with the consent of each patient and with institutional research ethics committee approval.
Plasmids. To silence endogenous GINS2 expression, the following 2 short hairpin RNAs (shRNAs) were synthesized and purchased: GINS2 shRNA1, CCGGATCCCGAAGGCA GACGAAATCCTCGAGGATTTCGTCTGCCTTCGGGAT TTTTTG; GINS2 shRNA2, CCGGGAATGGATTCAGGAT GTTGTTCTCGAGAACAACATCCTGAATCCATTCTTT TTG. The shRNA sequences were cloned into pSuper-retro-neo plasmids to generate the respective pSuper-retro-GINS2RNAi(s). After $48 \mathrm{~h}$ infection, the SiHa and HeLa cell lines stably expressing the GINS2 shRNAs were selected with puromycin $(0.5 \mu \mathrm{g} / \mathrm{ml})$.

Real-time PCR. Total RNA from cervical cancer cells and fresh tumor tissues was extracted using TRIzol (Invitrogen, Carlsbad, CA, USA) according to the manufacturer's instructions. The isolated RNA was pretreated with RNase-free DNase, and $2 \mu \mathrm{g}$ RNA/sample was used for complementary DNA (cDNA) synthesis. For the PCR amplication of GINS2 cDNA, an initial amplification step using CINS2-specific primers was performed with denaturation at $95^{\circ} \mathrm{C}$ for $10 \mathrm{~min}$, followed by 28 denaturation cycles at $95^{\circ} \mathrm{C}$ for $60 \mathrm{sec}$, primer annealing at $58^{\circ} \mathrm{C}$ for $30 \mathrm{sec}$, and a primer extension phase at $72^{\circ} \mathrm{C}$ for $30 \mathrm{sec}$. Upon completion of the cycling steps, a final extension step at $72^{\circ} \mathrm{C}$ for $5 \mathrm{~min}$ was performed before the reaction mixture was stored at $4^{\circ} \mathrm{C}$. Quantitative PCR (qPCR) was then conducted to determine the increase in GINS2 mRNA in each of the primary cervical tumors relative to the paired normal cervical tissue from the same patient and in the 8 cervical cancer cell lines relative to that in normal cervical tissue. Expression data were normalized to the geometric mean of the expression level of the housekeeping gene glyceraldehyde-3-phosphate dehydrogenase (GAPDH). The primers were designed using Primer Express v 2.0 software (Applied Biosystems, Foster City, CA, USA). The GINS2 forward and reverse primer sequences were: 5'-GCTGGCGATTAAC CTGAAAC-3' and 5'-TTCCTTTCGTTCATGATCCC-3', respectively. The GAPDH forward and reverse primers were: 5'-TTGAGGTCAATGAAGGGGTC-3' and 5'-GAAGGTGAA GGTCGGAGTCA-3', respectively.

Western blotting. Cells at 80-90\% confluence were washed twice with ice-cold phosphate-buffered saline (PBS) and lysed on ice in radioimmunoprecipitation assay buffer (RIPA; Cell Signaling Technology, Danvers, MA, USA) containing complete protease inhibitor cocktail (Roche Applied Sciences, Mannheim, Germany). Fresh tissue samples were ground to powder in liquid nitrogen and lysed with SDS-PAGE sample buffer. Protein concentration was determined by the Bradford assay (Bio-Rad Laboratories, Hercules, CA, USA). Equal protein samples $(30 \mu \mathrm{g})$ extracted from the cervical cancer cell lines and tissues were electrophoretically separated on $10.5 \%$ sodium dodecyl sulfate (SDS)/polyacrylamide gels, and transferred onto polyvinylidene difluoride (PVDF) membranes (Immobilon-P; Millipore, Bedford, MA, USA). The membranes were blocked with $5 \%$ fat-free milk in Tris-buffered saline containing 0.1\% Tween-20 (TBST) for $1 \mathrm{~h}$ at room temperature. The membranes were then incubated with anti-GINS2 rabbit monoclonal antibody (1:2,000; HPA057285; Sigma, St. Louis, MO, USA) overnight at $4^{\circ} \mathrm{C}$. After washing 3 times with TBST, the membranes 
Table I. Clinicopathological features and GINS2 expression of patients $(n=155)$ with early-stage cervical cancer.

\begin{tabular}{|c|c|}
\hline Characteristics & No. of cases $(C$ \\
\hline \multicolumn{2}{|l|}{ Age (years) } \\
\hline$\leq 47$ & $78(50.3)$ \\
\hline$>47$ & $77(49.7)$ \\
\hline \multicolumn{2}{|l|}{ FIGO stage } \\
\hline Ia & $18(11.6)$ \\
\hline $\mathrm{Ib}$ & $83(53.5)$ \\
\hline IIa & $54(34.9)$ \\
\hline \multicolumn{2}{|l|}{ Histological type } \\
\hline Squamous cell carcinoma & $149(96.1)$ \\
\hline Adenocarcinoma & $6(3.9)$ \\
\hline \multicolumn{2}{|c|}{ Pelvic lymph node metastasis (PLNM) } \\
\hline No & $99(63.9)$ \\
\hline Yes & $56(36.1)$ \\
\hline \multicolumn{2}{|l|}{ Tumor size $(\mathrm{cm})$} \\
\hline$\leq 4$ & $132(85.2)$ \\
\hline$>4$ & $23(14.8)$ \\
\hline \multicolumn{2}{|l|}{ SCC-Ag (ng/ml) } \\
\hline$\leq 1.5$ & $91(58.7)$ \\
\hline$>1.5$ & $64(41.3)$ \\
\hline \multicolumn{2}{|l|}{ Deep stromal invasion } \\
\hline No & 49 (31.6) \\
\hline Yes & $106(68.4)$ \\
\hline \multicolumn{2}{|c|}{ Lymphovascular space involvement } \\
\hline No & $146(94.2)$ \\
\hline Yes & $9(5.8)$ \\
\hline \multicolumn{2}{|l|}{ Positive surgical margin } \\
\hline No & $149(96.1)$ \\
\hline Yes & $6(3.9)$ \\
\hline \multicolumn{2}{|l|}{ Positive parametrium } \\
\hline No & $149(96.1)$ \\
\hline Yes & $6(3.9)$ \\
\hline \multicolumn{2}{|l|}{ Tumor recurrence } \\
\hline No & $137(88.4)$ \\
\hline Yes & $18(11.6)$ \\
\hline \multicolumn{2}{|l|}{ Vital status (at follow-up) } \\
\hline Alive & $126(81.3)$ \\
\hline Dead & $29(18.7)$ \\
\hline \multicolumn{2}{|l|}{ Expression of GINS2 } \\
\hline Low or no expression & $48(31.0)$ \\
\hline High expression & $107(69.0)$ \\
\hline
\end{tabular}

GINS2, complex subunit 2; SCC-Ag, squamous cell carcinoma antigen; FIGO, International Federation of Obstetrics and Gynecology.

were probed with horseradish peroxidase-conjugated goat anti-rabbit immunoglobulin G (1:2,000; SC-2004; Santa Cruz Biotechnology, Santa Cruz, CA, USA), and protein expression was detected by enhanced chemiluminescence (Amersham Pharmacia Biotech, Piscataway, NJ, USA) according to the

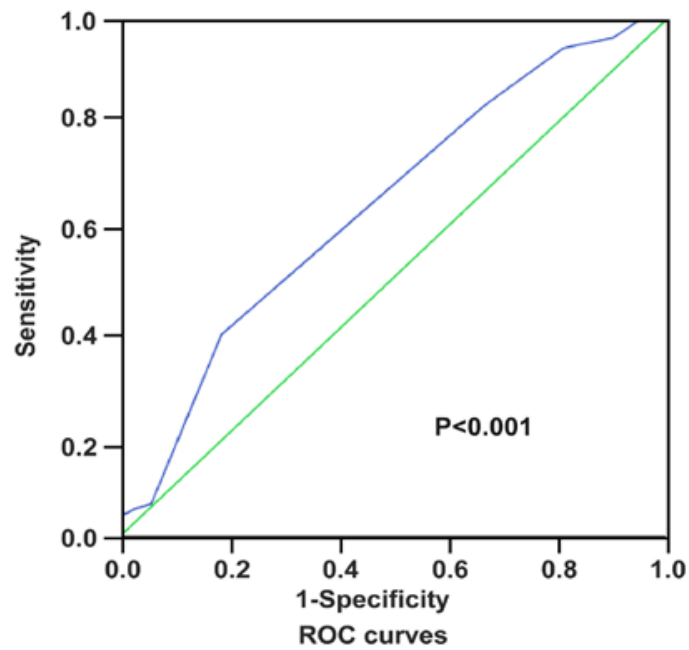

Figure 1. Receiver operating characteristics (ROC) curve analysis to determine the optimal cut-off values for IHC staining index scores and 7 was defined as the cut-off point. Accordingly, scores $\geq 8$ were judged as high GINS2 expression and scores $<8$ were categorized as low GINS2 expression.

manufacturer's suggested protocols. An anti- $\alpha$-tubulin mouse monoclonal or an anti-GAPDH mouse monoclonal antibody (1:2,000; Sigma) was used as a loading control.

Immunohistochemistry (IHC). IHC was used to examine GINS2 expression in 155 human cervical cancer specimens. Briefly, the paraffin-embedded specimens were cut into $4-\mu \mathrm{m}$ sections and baked at $60^{\circ} \mathrm{C}$ for $1 \mathrm{~h}$, deparaffinized with xylenes and rehydrated, submerged in EDTA antigen retrieval buffer, and microwaved for antigen retrieval. The samples were treated with $3 \%$ hydrogen peroxide in methanol to quench the endogenous peroxidase activity, followed by incubation with $1 \%$ bovine serum albumin to block non-specific binding. The sections were then incubated with anti-GINS2 rabbit monoclonal antibody (1:600; HPA057285; Sigma) overnight at $4^{\circ} \mathrm{C}$. Normal goat serum was used as the negative control. After washing, the sections were incubated with a biotinylated anti-rabbit secondary antibody, followed by incubation with streptavidin-horseradish peroxidase complex (both from Abcam, Cambridge, MA, USA). The sections were immersed in 3-amino-9-ethylcarbazole and counterstained with $10 \%$ Mayer's hematoxylin, dehydrated and mounted in crystal mount.

Two independent observers blinded to the histopathological features and patient data of the samples evaluated and scored the degree of immunostaining. The scores were based on the proportion of positively stained tumor cells [graded as: 1 ( $<10 \%$ positive), 2 (10-50\% positive), 3 (51-75\% positive) or $4(>75 \%$ positive)] and staining intensity [categorized as 1 (no staining), 2 (weak staining, light yellow), 3 (moderate staining, yellow brown) or 4 (strong staining, brown)]. The staining index was generated by multiplying the scores for staining intensity and for the proportion of positive cells (scored as 1 , $2,3,4,6,8,9,12$ and 16). Staining index score $\geq 8$ indicated tumors with high GINS2 expression; a score of $<8$ defined low GINS2 expression. Receiver operating characteristics (ROC) curve analysis was conducted using variables including GINS2 expression and patient outcomes to determine the optimum cut-off values of the scores (Fig. 1). 
Colony formation and anchorage-independent growth ability

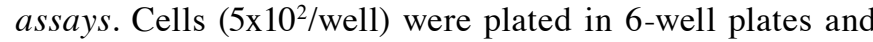
cultured for 2 weeks. The colonies were washed with PBS 3 times and fixed with $4 \%$ formaldehyde for $10 \mathrm{~min}$. Then, the colonies were stained with $1 \%$ crystal violet for $10 \mathrm{~min}$. After washing, the colonies were counted. For the anchorage-independent growth ability assay, 500 cells were trypsinized and suspended in $2 \mathrm{ml}$ complete medium plus $0.3 \%$ agar (Sigma). The agar-cell mixture was plated on $1 \%$ agar complete medium mixture. For $\sim 10$ days, viable colonies that were $>0.1 \mathrm{~mm}$ in diameter were counted. The experiment was carried out in triplicate for each cell line.

Wound healing and Transwell migration and invasion assays. In the wound healing assay, cells $\left(2 \times 10^{6} /\right.$ well) were seeded in 6 -well plates. When the cells were $90 \%$ confluent, they were serum-starved for $24 \mathrm{~h}$. A linear wound was created in the confluent monolayer using a $10-\mu l$ pipette tip. The wounds were observed and photographed immediately (time 0) and thereafter at 24 and $48 \mathrm{~h}$ (magnification, x200). Each experiment was repeated at least 3 times. For the Transwell migration and invasion assays, $2 \times 10^{4}$ cells were seeded in $8-\mu \mathrm{m}$ pore inserts coated with (for invasion) or without (for migration) $50 \mu \mathrm{l}$ Matrigel in triplicate wells. After $24 \mathrm{~h}$ incubation at $37^{\circ} \mathrm{C}$, cells that had passed through the filter into the bottom chamber were fixed with $1 \%$ paraformaldehyde, stained with hematoxylin, and counted under a magnification of x 200 (10 random fields/well).

Statistical analysis. All statistical analyses were conducted using the SPSS software package (standard version 16.0; IBM). The Chi-square and Fisher's exact tests were used to evaluate the relationship between GINS2 expression and the clinicopathological features. Spearman's rank correlation coefficients were performed to calculate bivariate correlations between the study variables. Survival curves were estimated using the Kaplan-Meier method and the log-rank test. The independent prognostic indicator in all of the clinical parameters was determined using univariate and multivariate analyses. $\mathrm{P}<0.05$ was considered statistically significant in all cases.

\section{Results}

GINS2 is overexpressed in cervical cancer. We used real-time PCR and western blotting to determine the GINS2 expression pattern in cervical carcinoma cell lines and samples. There were higher levels of both GINS2 transcription and translation in the cervical carcinoma cell lines in comparison to normal cervical tissue (Fig. 2A). Consistent with the findings in the cervical cancer cell lines, GINS2 mRNA and protein levels were clearly differentially increased in the 8 cervical cancer tissue samples than these levels in the matched adjacent noncancerous tissues (Fig. 2B). Furthermore, IHC confirmed GINS2 overexpression in the cervical cancer clinical samples (Fig. 2C).

GINS2 expression correlates with clinical characteristics in early-stage cervical cancer. To investigate the clinical relevance of GINS2 expression and cervical cancer progression, IHC was performed on 155 paraffin-embedded, archived clinical cervical cancer samples, which included 18, 83 and 54 cases of stage Ia 2 , Ib and IIa disease, respectively. We detected strong positive expression of GINS2 in 48 (31\%) cervical cancer specimens, whereas there was no or marginally detectable staining in the remaining 107 (69\%; Table I) clinical tumor samples. GINS2 primarily localized in the tumor cell nuclei and was absent from the adjacent normal cervical tissues (Fig. 3).

Notably, GINS2 expression correlated with several clinical features of cervical cancer, including SCC-Ag $(\mathrm{P}<0.001)$, pelvic LNM ( $\mathrm{PLNM}$; $\mathrm{P}<0.001)$, deep stromal invasion $(\mathrm{P}=0.021)$, vital status $(\mathrm{P}<0.001)$ and recurrence $(\mathrm{P}<0.001)$. In contrast, GINS2 and other clinical characteristics, including FIGO stage, surgical margin, lymphovascular invasion, parauterine organ infiltration and age, were not obviously related (Table II). Spearman correlation analysis (Table III) further confirmed the strong association of GINS2 expression and significant prognostic risk factors, including $\mathrm{SCC}-\mathrm{Ag}(\mathrm{R}=0.430$; $\mathrm{P}<0.001)$, deep stromal invasion $(\mathrm{R}=0.185 ; \mathrm{P}=0.021)$, vital status $(\mathrm{R}=0.287 ; \mathrm{P}<0.001)$, recurrence $(\mathrm{R}=0.323 ; \mathrm{P}<0.001)$, and in particular, $\mathrm{PLNM}(\mathrm{R}=0.455 ; \mathrm{P}<0.001)$.

GINS2 expression is associated with poor survival outcomes in early-stage cervical cancer. We used Kaplan-Meier survival curves and the log-rank test to evaluate the impact of GINS2 on predicting the prognosis of patients with early-stage cervical cancer. Fig. 4A shows that patients with higher levels of GINS2 had shorter OS $(\mathrm{P}<0.001)$, whereas those with lower GINS2 expression survived longer. For the patients with low GINS2 expression, the cumulative OS rates were $89.36 \%$ [95\% confidence interval (CI), 85.68-93.05\%], but were drastically decreased to $65.19 \%$ (95\% CI, 53.89-76.49\%) in the high GINS2 group. In addition, we calculated the prognostic value of GINS2 expression in specific patient subgroups, which were stratified based on age, FIGO stage, PLNM, SCC-Ag, tumor size, differentiation grade, deep stromal invasion, properties of the surgical margin, parauterine organ infiltration and lymphovascular space involvement. Fig. 4B-D shows that there was a markedly negative association between GINS2 expression and OS in the patients without LNM (log-rank test; $\mathrm{P}<0.001$ ), with SCC-Ag $>1.5 \mathrm{ng} / \mathrm{ml}$ (log-rank test; $\mathrm{P}=0.0019$ ), and with deep stromal invasion (log-rank test; $\mathrm{P}<0.001)$. The Cox regression model indicated that GINS2 expression and recurrence were independent prognostic risk factors of cervical cancer (Table IV). Taken together, our results indicate that GINS2 plays an important role in cervical cancer progression and may serve as a valuable prognostic predictor for patients with early-stage cervical cancer.

GINS2 downregulation inhibits cervical cancer cell proliferation and tumorigenic ability. Given the involvement of GINS2 in cervical cancer progression and its potential as a biomarker for identifying patients with a more aggressive cervical cancer phenotype, we explored the effects of GINS2 on the proliferation and tumorigenicity of cervical cancer cell lines. Two shRNAs against GINS2 were transduced into the SiHa and HeLa cervical cancer cell lines to stably suppress endogenous GINS2 expression. The downregulation efficiency was verified using western blotting (Fig. 5A). As expected, the colony formation assay revealed that GINS2 
A

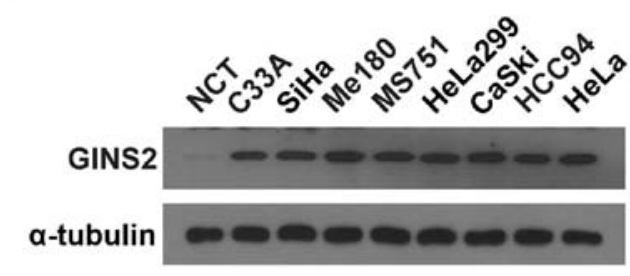

B

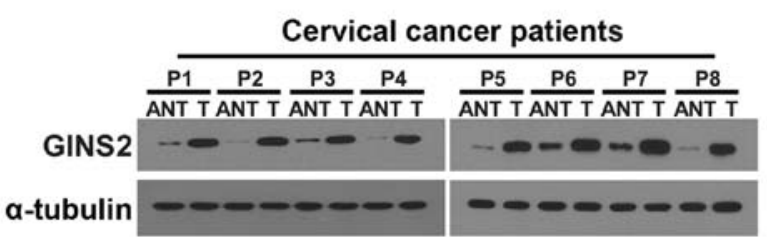

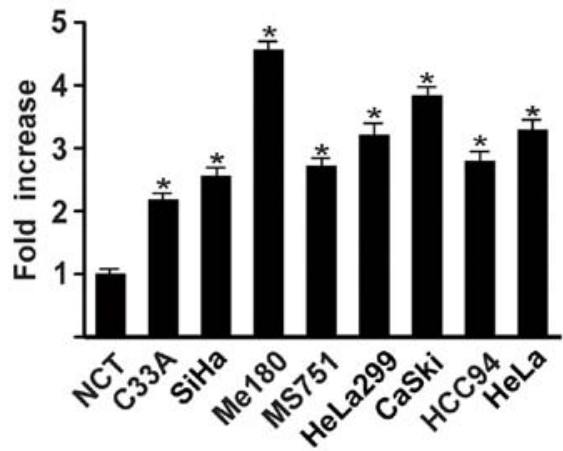

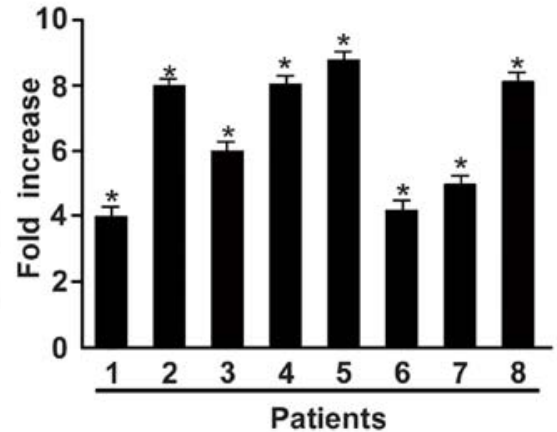

C
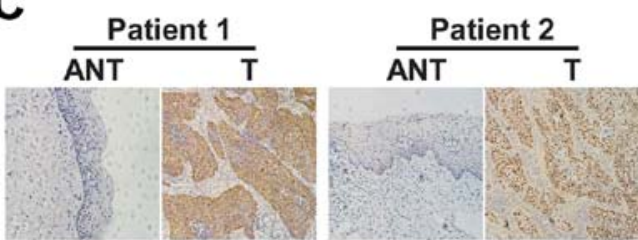

$\frac{\text { Patient } 3}{\text { ANT }}$
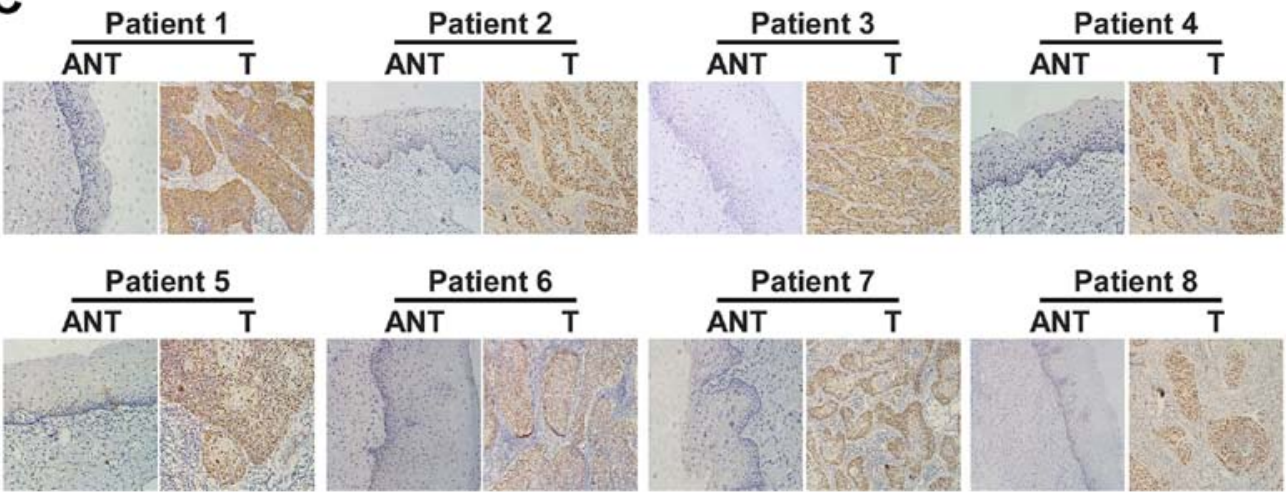

Figure 2. Western blotting, qPCR, and IHC determination of GINS2 mRNA and protein expression. (A) Western blotting and qPCR of GINS2 expression in normal cervical tissues and cervical cancer cell lines. (B) Western blotting of GINS2 expression in 8 pairs of matched cervical cancer (T) and adjacent noncancerous cervical tissues (ANT) and average T/ANT ratios of GINS2 mRNA expression quantified by qPCR in 8 pairs of matched cervical cancer tissues. Expression levels were normalized to $\alpha$-tubulin expression. Error bars represent standard deviation (SD) calculated from 3 parallel experiments. " $\mathrm{P}<0.05$. (C) IHC assay of GINS2 protein expression in 8 pairs of matched cervical cancer tissues.

A

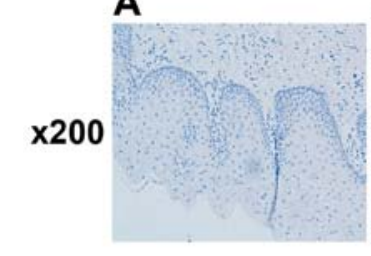

B
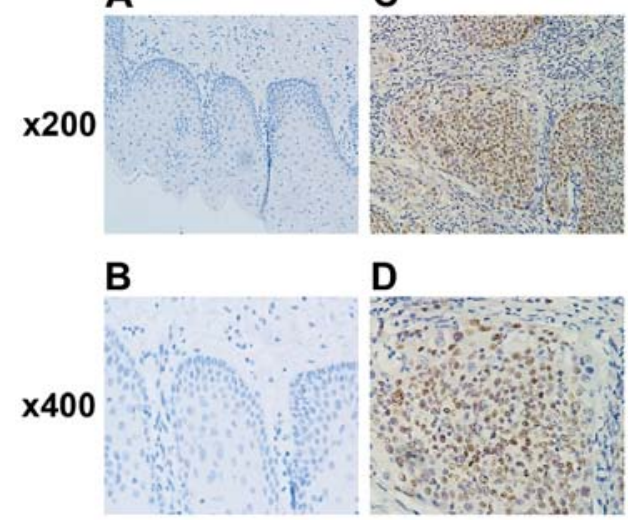

E

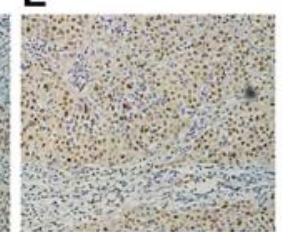

$\mathbf{F}$

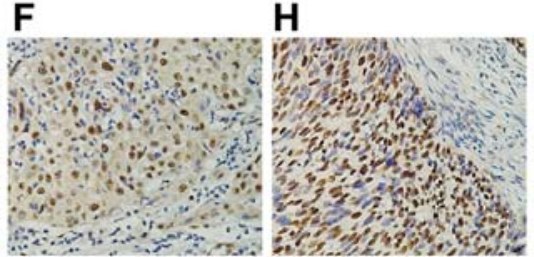

Figure 3. IHC assay of GINS2 expression in cervical cancer tissues. Original magnification x200 or x400. Positive GINS2 staining was observed mainly in cervical cancer cell nuclei. (A and B) GINS2 was not detected in normal cervical cancer tissues. (C and D) Representative images of weak GINS2 staining in cervical cancer tissues. (E and F) Representative images of moderate GINS2 staining in cervical cancer tissues. (G and H) Representative images of strong GINS2 staining in cervical cancer tissues. 
Table II. Correlation of clinicopathological characteristics and GINS2 expression in the early-stage cervical cancer patients.

\begin{tabular}{|c|c|c|c|c|c|}
\hline \multirow[b]{2}{*}{ Characteristics } & \multirow[b]{2}{*}{$\begin{array}{c}\text { Total } \\
(\mathrm{n}=155)\end{array}$} & \multicolumn{2}{|c|}{ GINS2 } & \multirow[b]{2}{*}{$\begin{array}{c}\text { Chi-square test } \\
\text { P-value }\end{array}$} & \multirow[b]{2}{*}{$\begin{array}{c}\text { Fisher's exact test } \\
\text { P-value }\end{array}$} \\
\hline & & $\begin{array}{c}\text { Low } \\
\text { expression }\end{array}$ & $\begin{array}{c}\text { High } \\
\text { expression }\end{array}$ & & \\
\hline Age (years) & & & & 0.688 & 0.730 \\
\hline$\leq 47$ & 78 & $55(35.5)$ & $23(14.8)$ & & \\
\hline$>47$ & 77 & $52(33.5)$ & $25(16.2)$ & & \\
\hline FIGO stage & & & & 0.571 & \\
\hline Ia & 18 & $14(9.0)$ & $4(2.6)$ & & \\
\hline $\mathrm{Ib}$ & 83 & $58(37.4)$ & $25(16.1)$ & & \\
\hline IIa & 54 & $35(22.6)$ & $19(12.3)$ & & \\
\hline Pelvic lymph node metastasis (PLNM) & & & & $<0.001$ & $<0.001$ \\
\hline No & 99 & $84(54.2)$ & $15(9.7)$ & & \\
\hline Yes & 56 & $23(14.8)$ & $33(21.3)$ & & \\
\hline Histological types & & & & 0.440 & 0.667 \\
\hline Squamous cell carcinoma & 149 & $102(65.8)$ & $47(30.3)$ & & \\
\hline Adenocarcinoma & 6 & $5(3.2)$ & $1(0.7)$ & & \\
\hline SCC-Ag (ng/ml) & & & & $<0.001$ & $<0.001$ \\
\hline$\leq 1.5$ & 91 & $78(50.3)$ & $13(8.4)$ & & \\
\hline$>1.5$ & 64 & $29(18.7)$ & $35(22.6)$ & & \\
\hline Tumor size (cm) & & & & 0.058 & 0.085 \\
\hline$\leq 4$ & 132 & $95(61.3)$ & $37(23.9)$ & & \\
\hline$>4$ & 23 & $12(7.7)$ & $11(7.1)$ & & \\
\hline Positive surgical margin & & & & 0.304 & 0.374 \\
\hline No & 149 & $104(67.1)$ & $45(29.0)$ & & \\
\hline Yes & 6 & $3(1.9)$ & $3(1.9)$ & & \\
\hline Deep stromal invasion & & & & 0.021 & 0.025 \\
\hline No & 49 & $40(25.8)$ & $9(5.8)$ & & \\
\hline Yes & 106 & $67(43.2)$ & $39(25.2)$ & & \\
\hline Positive parametrium & & & & 0.898 & 1.000 \\
\hline No & 149 & $103(66.5)$ & $46(29.7)$ & & \\
\hline Yes & 6 & $4(2.6)$ & $2(1.3)$ & & \\
\hline Lymphovascular space involvement & & & & 0.874 & 1.000 \\
\hline No & 146 & $101(65.2)$ & $45(29.0)$ & & \\
\hline Yes & 9 & $6(3.9)$ & $3(1.9)$ & & \\
\hline Vital status (at follow-up) & & & & $<0.001$ & 0.001 \\
\hline Alive & 126 & $95(61.3)$ & $31(20.0)$ & & \\
\hline Dead & 29 & $12(7.7)$ & $17(11.0)$ & & \\
\hline Recurrence & & & & $<0.001$ & $<0.001$ \\
\hline No & 137 & $102(65.8)$ & $35(22.6)$ & & \\
\hline Yes & 18 & $5(3.2)$ & $13(8.4)$ & & \\
\hline
\end{tabular}

GINS2, complex subunit 2; SCC-Ag, squamous cell carcinoma antigen; FIGO, International Federation of Obstetrics and Gynecology.

depletion induced the formation of much smaller and fewer colonies as compared to the vector control cells (Fig. 5B). Furthermore, silencing of GINS2 markedly decreased the anchorage-independent growth ability of the two cell lines in soft agar (Fig. 5C). These results suggest that GINS2 promotes proliferation and tumorigenesis of cervical cancer cells.
GINS2 inhibition reduces cervical cancer cell migration and invasion. After demonstrating that GINS2 is closely connected with PLNM, we then investigated whether GINS2 regulates cervical cancer cell motility and invasiveness. Fig. 6A shows that in the wound healing assay, GINS2 ablation slowed the speed with which the SiHa and HeLa cells filled 

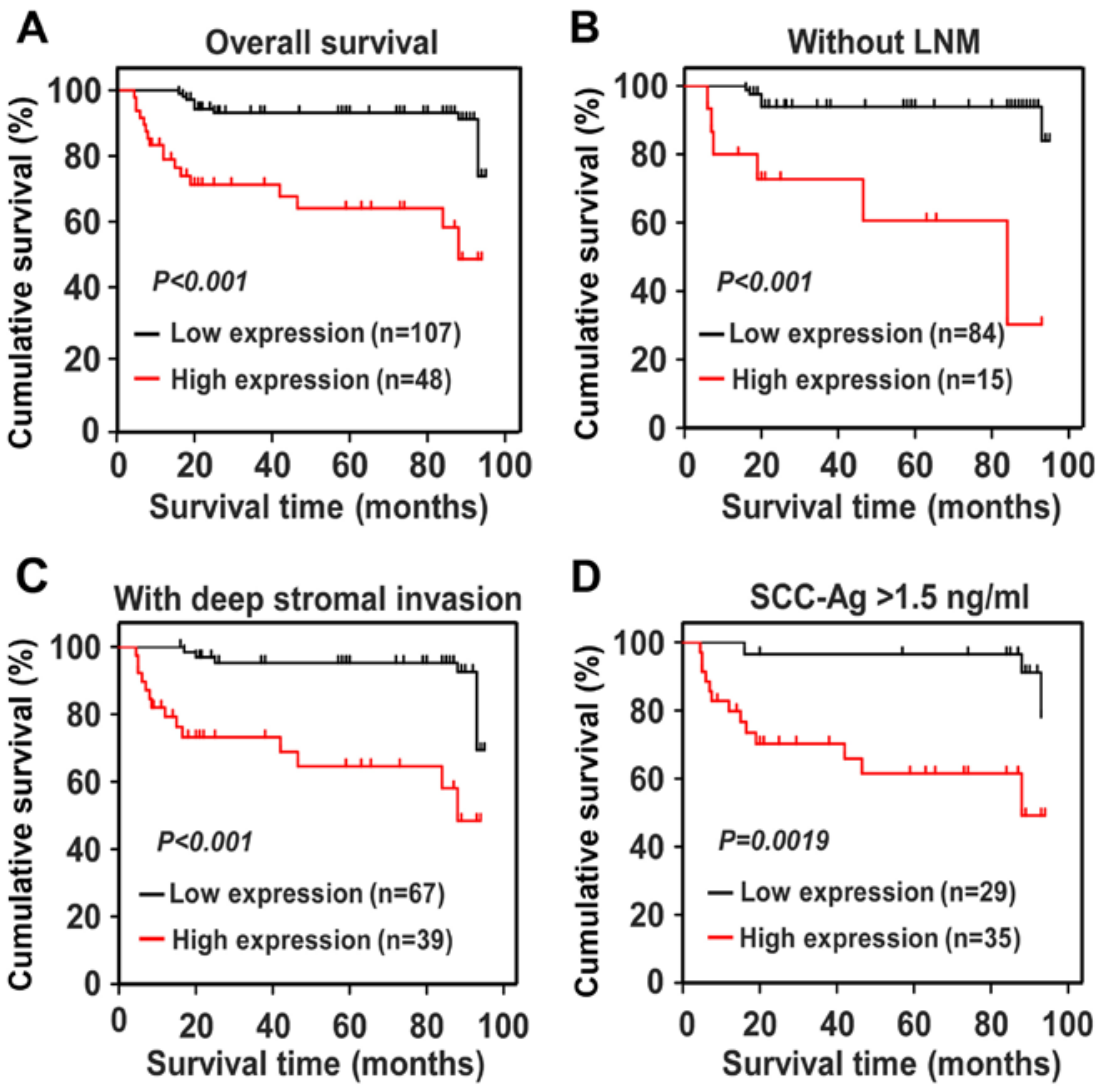

Figure 4. Kaplan-Meier curves of univariate analysis data (log-rank test). (A) OS curves of patients with high vs. low GINS2 expression. (B) OS curves of patients without LNM with high vs. low GINS2 expression. (C) OS curves of patients with deep stromal invasion with high vs. low GINS2 expression. (D) OS curves of patients with SCC-Ag $>1.5 \mathrm{ng} / \mathrm{ml}$ with high vs. low GINS2 expression.

Table III. Spearman analysis of the correlation of GINS2 expression and clinicopathological features.

\begin{tabular}{lcr}
\hline & \multicolumn{2}{c}{ GINS2 expression level } \\
\cline { 2 - 3 } Variables & $\begin{array}{c}\text { Spearman } \\
\text { correlation }\end{array}$ & P-value \\
\hline PLNM & 0.455 & $<0.001$ \\
SCC-Ag (ng/ml) & 0.430 & $<0.001$ \\
Vital status (at follow-up) & 0.287 & $<0.001$ \\
Deep stromal invasion & 0.185 & 0.021 \\
Recurrence & 0.323 & $<0.001$ \\
\hline
\end{tabular}

GINS complex subunit 2; PLNM, pelvic lymph node metastasis; $\mathrm{SCC}-\mathrm{Ag}$, squamous cell carcinoma antigen.

the gap in comparison to the control in an obvious manner. The Transwell migration and matrix invasion assays yielded similar results (Fig. 6B and C), as indicated by the smaller number of migrated and invaded cells that passed through the filter in the GINS2 downregulation group as compared to the negative control group. Collectively, our findings confirmed that GINS2 enhances the migratory and invasive properties of cervical cancer cells.

\section{Discussion}

In the present study, we demonstrated for the first time that GINS2 upregulation correlated with poor prognosis and reduced survival in early-stage cervical cancer. In addition, GINS2 downregulation hampered the cellular capabilities of proliferation, tumorigenesis, migration and invasion in an obvious manner. These findings are convincing evidence that GINS2 plays a significant role in the progression of cervical cancer and has the potential to be a neoteric prognostic biomarker of early-stage cervical cancer.

Clinical evidence has confirmed the role of GINS2 as an oncogene in various malignancies. GINS2 is elevated in intrahepatic cholangiocarcinoma, lung adenocarcinoma and breast cancer, and induces intrahepatic cholangiocarcinoma cell proliferation $(7,8,10)$. Moreover, GINS2 overexpression contributes to maintenance of the cancer stem cell population and has been correlated with progression and poor prognosis in breast cancer (8). The above mentioned studies indicated that GINS2 plays a critical role in carcinogenesis and cancer progression. Herein, we investigated its role in cervical cancer. Consistent with the above studies, GINS2 mRNA and protein expression was higher in the 8 cervical cancer cell lines and clinical samples. IHC analysis indicated that GINS2 expression was associated with well-known prognostic parameters: SCC-Ag $(\mathrm{P}<0.001)$, deep stromal invasion $(\mathrm{P}=0.021)$, vital status $(\mathrm{P}<0.001)$ and recurrence $(\mathrm{P}<0.001)$, particularly PLNM $(\mathrm{P}<0.001)$, providing strong evidence that GINS2 
Table IV. Univariate and multivariate analysis of the prognostic parameters in early-stage cervical cancer using Cox-regression model.

\begin{tabular}{|c|c|c|c|c|}
\hline & \multicolumn{2}{|c|}{ Univariate analysis } & \multicolumn{2}{|c|}{ Multivariate analysis } \\
\hline & P-value & $\begin{array}{l}\text { Hazard ratio } \\
(95 \% \mathrm{CI})\end{array}$ & P-value & $\begin{array}{l}\text { Hazard ratio } \\
(95 \% \mathrm{CI})\end{array}$ \\
\hline $\begin{array}{l}\text { GINS2 expression } \\
\text { Low } \\
\text { High }\end{array}$ & $<0.001$ & $\begin{array}{c}5.160 \\
(2.438-10.925)\end{array}$ & 0.011 & $\begin{array}{c}3.643 \\
(1.347-9.850)\end{array}$ \\
\hline $\begin{array}{l}\text { Pelvic lymph node metastasis (PLNM) } \\
\text { No } \\
\text { Yes }\end{array}$ & 0.008 & $\begin{array}{c}2.716 \\
(1.299-5.680)\end{array}$ & 0.127 & $\begin{array}{c}1.827 \\
(0.843-3.962)\end{array}$ \\
\hline $\begin{array}{l}\mathrm{SCC}-\mathrm{Ag}(\mathrm{ng} / \mathrm{ml} \\
\leq 1.5 \\
>1.5\end{array}$ & 0.099 & $\begin{array}{c}1.854 \\
(0.891-3.855)\end{array}$ & 0.862 & $\begin{array}{c}0.922 \\
(0.369-2.303)\end{array}$ \\
\hline $\begin{array}{l}\text { Recurrence } \\
\text { No } \\
\text { Yes }\end{array}$ & $<0.001$ & $\begin{array}{c}10.201 \\
(4.883-21.352)\end{array}$ & $<0.001$ & $\begin{array}{c}8.523 \\
(3.944-18.416)\end{array}$ \\
\hline $\begin{array}{l}\text { Deep stromal invasion } \\
\text { No } \\
\text { Yes }\end{array}$ & 0.543 & $\begin{array}{c}1.304 \\
(0.555-3.063)\end{array}$ & 0.669 & $\begin{array}{c}0.823 \\
(0.337-2.008)\end{array}$ \\
\hline $\begin{array}{l}\text { Positive parametrium } \\
\text { No } \\
\text { Yes }\end{array}$ & 0.840 & $\begin{array}{c}1.228 \\
(0.166-9.092)\end{array}$ & 0.510 & $\begin{array}{c}2.076 \\
(0.236-18.222)\end{array}$ \\
\hline $\begin{array}{l}\text { Lymphovascular space } \\
\text { involvement }\end{array}$ & 0.735 & 1.282 & 0.971 & 1.032 \\
\hline $\begin{array}{l}\text { No } \\
\text { Yes }\end{array}$ & & $(0.304-5.400)$ & & $(0.190-5.623)$ \\
\hline $\begin{array}{l}\text { Tumor size }(\mathrm{cm}) \\
\leq 4 \\
>4\end{array}$ & 0.523 & $\begin{array}{c}1.371 \\
(0.521-3.609)\end{array}$ & 0.481 & $\begin{array}{c}0.675 \\
(0.227-2.013)\end{array}$ \\
\hline
\end{tabular}

CI, confidential interval; GINS2, complex subunit 2; SCC-Ag, squamous cell carcinoma antigen.

plays an oncogenic role in cervical cancer development and that it may act as a biomarker for assessing patients with a more aggressive form of the disease. More importantly, the present study demonstrated that GINS2 overexpression correlates with poor clinical outcome and is potentially an independent prognostic factor of poor OS in cervical cancer. Taken together, our results implicate GINS2 as a crucial contributing factor in tumor progression.

Clearly, the fundamental characteristic of tumor cells is their malignant uncontrolled growth, the key process of which is DNA replication $(11,12)$. Correct DNA replication requires the proper combination of replication-associated proteins and prereplication complexes. Defects in the proper function of these proteins are responsible for chromosome instability and the subsequent generation of neoplasms (13-16). Indeed, aberrant expression of several DNA replication-related proteins has been demonstrated in certain cancers. Gan et al proposed MCM genes, which represent primary helicase for unwinding DNA during replication, as diagnostic or prognostic markers in cervical carcinoma (17). Tane et al showed that elevated levels of PSF3, a GINS family member, predicted poor prognosis of non-small cell lung cancer (18). In addition, silencing of PSF3 inhibited cell proliferation by inducing G1-S arrest, suggesting that PSF3 may be required for the development of lung cancer. In accordance with these previous studies, we established the clinical significance of GINS2 in cervical cancer and demonstrated that GINS2 knockdown markedly impaired the proliferative and tumorigenic properties of cervical cancer cells. GINS2 was previouly found to alter the percentages of the apoptosis-relevant genes BAX and BCL2, and the levels of the cell cycle regulators ataxia-telangiectasia mutated (ATM), checkpoint kinase 2 (CHK2), and P53 to stimulate G2/M transition (9). Accordingly, we similarly hypothesized that GINS2 accelerated G2/M transitional entry by circumventing cell cycle checkpoints, consequently exerting its oncogenic potential in cervical cancer. However, further investigations are needed to verify the hypothesis and to clarify the carcinogenesis mechanisms in greater detail. 


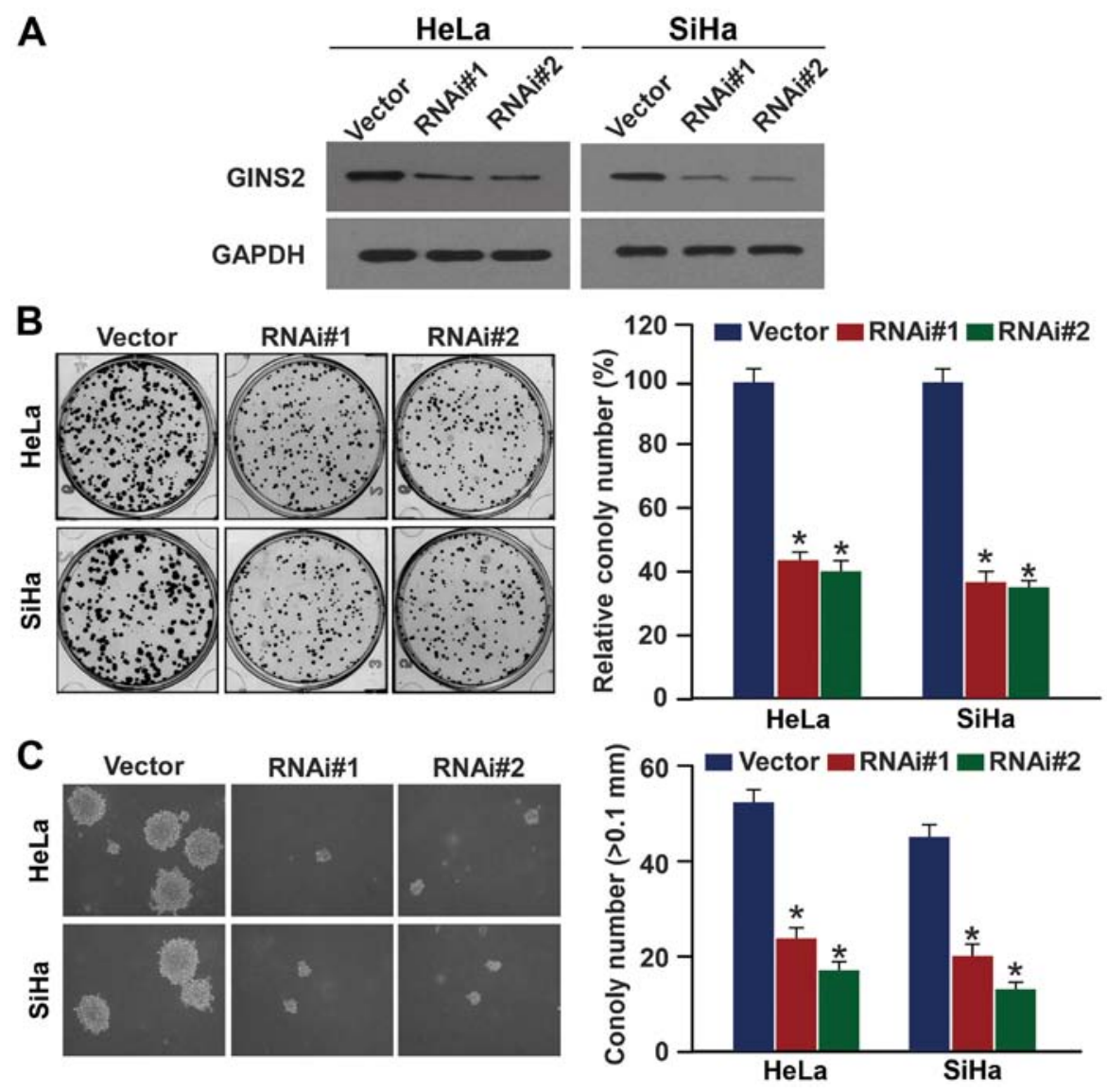

Figure 5. GINS2 promotes cervical cancer cell proliferation and tumorigenicity. (A) Western blot analysis of GINS2 expression in GINS2-silenced SiHa and HeLa cells. GAPDH was used as the loading control. (B) Colony formation assay showing that silencing of endogenous GINS2 inhibited proliferation. (C) Anchorage-independent growth ability assay showing that GINS2 knockdown suppressed cervical cancer cell tumorigenicity. The number of colonies $>0.1 \mathrm{~mm}$ in diameter was quantified after 10 days of culture. Original magnification, $\mathrm{x} 200$. Bars represent the mean $\pm \mathrm{SD}$ of 3 independent experiments; ${ }^{*} \mathrm{P}<0.05$.

Currently, radical hysterectomy plus pelvic lymph node dissection or chemoradiation is the criterion standard of treatment for patients with early-stage cervical cancer $(19,20)$. Lymph node status is critical for determining the appropriate treatment strategies. Patients with LNM require chemoradiation, which may render the initial surgical intervention unnecessary in retrospect $(19,21)$. Meanwhile, unnecessary lymphadenectomy in patients with negative LNM can cause unfavorable complications such as infection, lymphocysts, and lymphedema (22). However, imaging methods and sentinel lymph node biopsy both fail to accurately detect LNM $(21,23,24)$. Worse, there is no existing preoperative marker for sensitive and efficient LNM diagnosis. Surprisingly, our data showed that GINS2 was closely associated with LNM; moreover, inhibition of GINS2 significantly suppressed the aggressive cervical cancer cell phenotype. Therefore, we speculated that GINS2 is a valuable marker for predicting PLNM. However, a larger cohort of patients with LNM should be enrolled in future studies, and the definite regulatory mechanisms involved warrant further investigation.

SCC-Ag is currently most widely applied in clinical diagnosis, evaluation of therapeutic effects, and for predicting the clinical outcomes of patients with cervical cancer $(25,26)$. SCC-Ag levels are associated with the extent of cervical carcinoma (27-33). Sustained high or continuous elevation of SCC-Ag represents persistent disease or recurrence $(34,35)$. However, the clinical practical value of serum SCC-Ag is still controversial. The numerical changes of SCC-Ag do not specifically reflect cervical cancer; increased SCC-Ag levels have also been observed in patients with lung cancer, esophageal SCC and other benign diseases (36-38). More unfortunately, the autofit cut-off level of SCC-Ag is still a matter of debate partially due to the different disease stages of patient groups (39). Therefore, it appears that SCC-Ag is not an ideal indicator for predicting outcomes in cervical cancer.

Notably, we reported that GINS2 plays an important role in the development of cervical cancer and may serve as a valuable prognostic marker for assessing survival in cervical cancer. Therefore, determining the expression of GINS2 in cervical tissues via biopsy can provide significant guidance for deciding preferred treatment modality for patients with early-stage cervical cancer.

To date, persistent human papillomavirus (HPV) infection is recognized as the main cause of cervical cancer, and HPV 16 and HPV 18 are considered the most carcinogenic HPVs. The HPV oncoproteins E6 and E7 target the tumor-suppressor gene P53 and retinoblastoma protein ( $\mathrm{Rb})$, respectively, simultaneously with the activation of certain oncogenes and pathways, thereby coordinately contributing to the initiation 
A

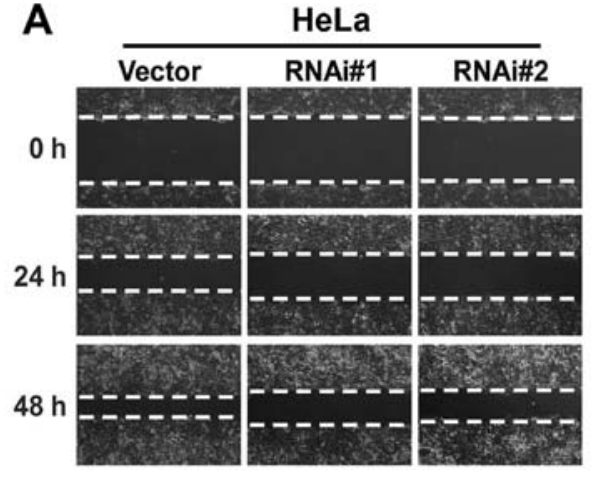

B

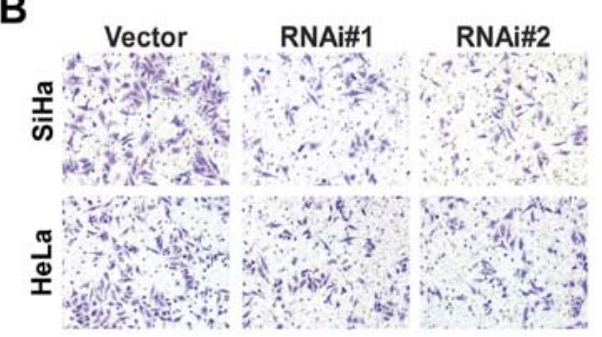

C

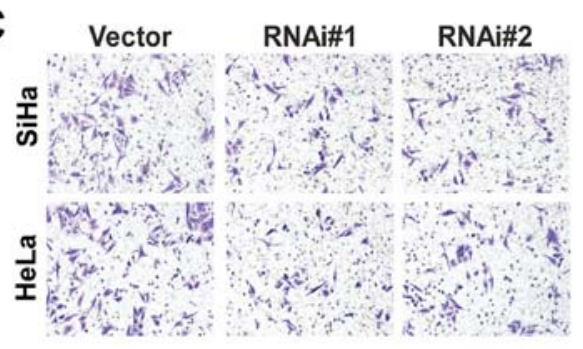

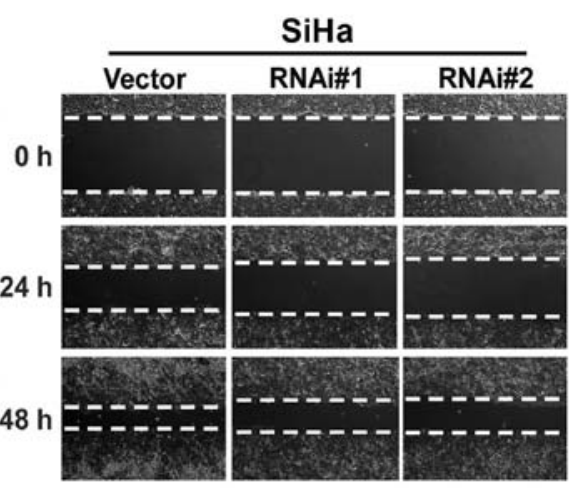
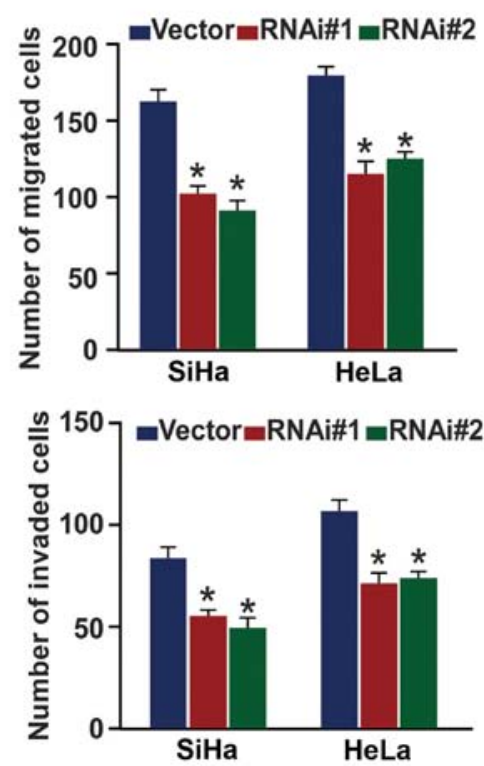

Figure 6. GINS2 is essential for cervical cancer cell migration and invasion. (A) Cell mobility was measured by examining the rate of wound closure at 0 , 24 and $48 \mathrm{~h}$. Original magnification, x200. (B) Transwell migration assay investigation of the mobility properties induced by fetal bovine serum. Original magnification, $\mathrm{x} 200 ;{ }^{*} \mathrm{P}<0.05$. (C) Transwell invasion assay of the invasive properties induced by fetal bovine serum. Original magnification, $\mathrm{x} 200$; ${ }^{*} \mathrm{P}<0.05$. Bars represent the mean \pm SD of 3 independent experiments

and progression of cervical cancer (40-42). Clinical evidence has demonstrated that GINS2 targets P53, and silencing of GINS2 upregulated P53 expression (9). We hypothesized that GINS2 cooperates with the HPV oncoprotein E6 jointly to promote tumor transformation and development. However, we did not investigate their interaction in the present study, and it warrants further investigation.

While the prognostic value of GINS2 in early-stage cervical cancer was validated in our research. However, there still existed limitations, one of which was the small sample size. Furthermore, as all the patients involved were all in the early-stage of cervical cancer, their prognosis was relatively favorable and few developed pathological risk features including LNM, positive parametrium, positive surgical margin, large tumor size and recurrence. Thus, to validate our results, it is urgent to incorporate a larger cohort of patients with aggressive clinical factors in our subsequent study.

In summary, the present study, confirms the aberrant expression and clinical significance of GINS2 in early-stage cervical cancer. Additionally, we found that GINS2 knockdown suppressed cervical cancer cell proliferation, tumorigenicity, migration and invasion. GINS2 may represent a novel indicator for identifying patients at high-risk, and potentially serve as a clinically relevant biomarker for predicting the patient outcome of early-stage cervical cancer, which could aid gynecologists in determining more appropriate therapeutic strategies.

\section{Acknowledgements}

The present study was funded by the Natural Science Foundation of Guangdong Province (S2013010015552), the National Natural Science Foundation of China (nos. 81602723, 81302550 and 81671805), the Postdoctoral Science Foundation of China (2016A020215214), and the Guangdong Medical Research Foundation (A2015130).

\section{References}

1. Jemal A, Bray F, Center MM, Ferlay J, Ward E and Forman D: Global cancer statistics. CA Cancer J Clin 61: 69-90, 2011.

2. Torre LA, Bray F, Siegel RL, Ferlay J, Lortet-Tieulent J and Jemal A: Global cancer statistics, 2012. CA Cancer J Clin 65: 87-108, 2015.

3. Noordhuis MG, Fehrmann RS, Wisman GB, Nijhuis ER, van Zanden JJ, Moerland PD, Ver Loren van Themaat E, Volders HH, Kok M, ten Hoor KA, et al: Involvement of the TGF-beta and beta-catenin pathways in pelvic lymph node metastasis in early-stage cervical cancer. Clin Cancer Res 17: 1317-1330, 2011. 
4. Yang L, Jia X, Li N, Chen C, Liu Y and Wang H: Comprehensive clinic-pathological characteristics of cervical cancer in southwestern China and the clinical significance of histological type and lymph node metastases in young patients. PLoS One 8 e75849, 2013.

5. Gambus A, Jones RC, Sanchez-Diaz A, Kanemaki M, van Deursen F, Edmondson RD and Labib K: GINS maintains association of $\mathrm{Cdc} 45$ with $\mathrm{MCM}$ in replisome progression complexes at eukaryotic DNA replication forks. Nat Cell Biol 8: 358-366, 2006.

6. Rantala JK, Edgren H, Lehtinen L, Wolf M, Kleivi K, Vollan HK, Aaltola AR, Laasola P, Kilpinen S, Saviranta P, et al: Integrative functional genomics analysis of sustained polyploidy phenotypes in breast cancer cells identifies an oncogenic profile for GINS2. Neoplasia 12: 877-888, 2010

7. Liu M, Pan H, Zhang F, Zhang Y, Zhang Y, Xia H, Zhu J, Fu W and Zhang X: Identification of TNM stage-specific genes in lung adenocarcinoma by genome-wide expression profiling. Oncol Lett 6: 763-768, 2013.

8. Zheng M, Zhou Y, Yang X, Tang J, Wei D, Zhang Y, Jiang JL, Chen ZN and Zhu P: High GINS2 transcript level predicts poor prognosis and correlates with high histological grade and endocrine therapy resistance through mammary cancer stem cells in breast cancer patients. Breast Cancer Res Treat 148 423-436, 2014

9. Zhang X, Zhong L, Liu BZ, Gao YJ, Gao YM and Hu XX: Effect of GINS2 on proliferation and apoptosis in leukemic cell line. Int J Med Sci 10: 1795-1804, 2013

10. Obama K, Ura K, Satoh S, Nakamura Y and Furukawa Y Up-regulation of PSF2, a member of the GINS multiprotein complex, in intrahepatic cholangiocarcinoma. Oncol Rep 14 701-706, 2005.

11. Mazurek A, Luo W, Krasnitz A, Hicks J, Powers RS and Stillman B: DDX5 regulates DNA replication and is required for cell proliferation in a subset of breast cancer cells. Cancer Discov 2: 812-825, 2012.

12. Costa A, Hood IV and Berger JM: Mechanisms for initiating cellular DNA replication. Annu Rev Biochem 82: 25-54, 2013.

13. Oehlmann M, Score AJ and Blow JJ: The role of Cdc6 in ensuring complete genome licensing and $\mathrm{S}$ phase checkpoint activation. J Cell Biol 165: 181-190, 2004

14. Weaver BA and Cleveland DW: Does aneuploidy cause cancer? Curr Opin Cell Biol 18: 658-667, 2006.

15. Dutta A: Chaotic license for genetic instability and cancer. Nat Genet 39: 10-11, 2007

16. Hermand D and Nurse P: Cdc18 enforces long-term maintenance of the S phase checkpoint by anchoring the Rad3-Rad26 complex to chromatin. Mol Cell 26: 553-563, 2007.

17. Gan N, Du Y, Zhang W and Zhou J: Increase of Mcm3 and Mcm4 expression in cervical squamous cell carcinomas. Eur J Gynaecol Oncol 31: 291-294, 2010.

18. Tane S, Sakai Y, Hokka D, Okuma H, Ogawa H, Tanaka Y, Uchino K, Nishio W, Yoshimura M and Maniwa Y: Significan role of Psf3 expression in non-small-cell lung cancer. Cancer Sci 106: 1625-1634, 2015.

19. Benedet JL, Bender H, Jones H III, Ngan HY and Pecorelli S; FIGO Committee on Gynecologic Oncology: FIGO staging classifications and clinical practice guidelines in the management of gynecologic cancers. Int J Gynaecol Obstet 70: 209-262, 2000.

20. Koh WJ, Greer BE, Abu-Rustum NR, Apte SM, Campos SM Chan J, Cho KR, Cohn D, Crispens MA, DuPont N, et al; National Comprehensive Cancer Network: Cervical cancer. J Natl Compr Canc Netw 11: 320-343, 2013.

21. Selman TJ, Mann C, Zamora J, Appleyard TL and Khan K: Diagnostic accuracy of tests for lymph node status in primary cervical cancer: A systematic review and meta-analysis. CMAJ 178: 855-862, 2008.

22. Matsuura Y, Kawagoe T, Toki N, Tanaka M and Kashimura M Long-standing complications after treatment for cancer of the uterine cervix - clinical significance of medical examination at 5 years after treatment. Int J Gynecol Cancer 6: 294-297, 2006.

23. Altgassen C, Hertel H, Brandstädt A, Köhler C, Dürst M and Schneider A; AGO Study Group: Multicenter validation study of the sentinel lymph node concept in cervical cancer: AGO Study Group. J Clin Oncol 26: 2943-2951, 2008.
24. Slama J, Dundr P, Dusek L and Cibula D: High false negative rate of frozen section examination of sentinel lymph nodes in patients with cervical cancer. Gynecol Oncol 129: 384-388, 2013.

25. Bonfrer JM, Gaarenstroom KN, Korse CM, Van Bunningen BN and Kenemans P: Cyfra 21-1 in monitoring cervical cancer: A comparison with tissue polypeptide antigen and squamous cell carcinoma antigen. Anticancer Res 17: 2329-2334, 1997.

26. Esajas MD, Duk JM, de Bruijn HW, Aalders JG, Willemse PH, Sluiter W, Pras B, ten Hoor K, Hollema H and van der Zee AG: Clinical value of routine serum squamous cell carcinoma antigen in follow-up of patients with early-stage cervical cancer. J Clin Oncol 19: 3960-3966, 2001.

27. Avall-Lundqvist EH, Sjovall K, Nilsson BR and Eneroth PH: Prognostic significance of pretreatment serum levels of squamous cell carcinoma antigen and CA 125 in cervical carcinoma. Eur J Cancer 28A: 1695-1702, 1992.

28. Duk JM, Groenier KH, de Bruijn HW, Hollema H, ten Hoor KA, van der Zee AG and Aalders JG: Pretreatment serum squamous cell carcinoma antigen: A newly identified prognostic factor in early-stage cervical carcinoma. J Clin Oncol 14: 111-118, 1996.

29. Yuan CC, Wang PH, Ng HT, Tsai LC, Juang CM and Chiu LM: Both TPA and SCC-Ag levels are prognostic even in high-risk stage Ib-IIa cervical carcinoma as determined by a stratification analysis. Eur J Gynaecol Oncol 23: 17-20, 2002.

30. Strauss HG, Laban C, Lautenschläger C, Buchmann J, Schneider I and Koelbl H: SCC antigen in the serum as an independent prognostic factor in operable squamous cell carcinoma of the cervix. Eur J Cancer 38: 1987-1991, 2002.

31. Molina R, Filella X, Lejarcegui JA, Pahisa J, Torné A, Rovirosa A, Mellado B, Ordi J, Puig-Tintore LM, Alicarte J, et al: Prospective evaluation of squamous cell carcinoma and carcinoembryonic antigen as prognostic factors in patients with cervical cancer. Tumour Biol 24: 156-164, 2003.

32. Reesink-Peters N, van der Velden J, Ten Hoor KA, Boezen HM, de Vries EG, Schilthuis MS, Mourits MJ, Nijman HW Aalders JG, Hollema H, et al: Preoperative serum squamous cell carcinoma antigen levels in clinical decision making for patients with early-stage cervical cancer. J Clin Oncol 23: 1455-1462, 2005.

33. Gaarenstroom KN, Kenter GG, Bonfrer JM, Korse CM, Van de Vijver MJ, Fleuren GJ and Trimbos JB: Can initial serum cyfra 21-1, SCC antigen, and TPA levels in squamous cell cervical cancer predict lymph node metastases or prognosis? Gynecol Oncol 77: 164-170, 2000.

34. Montag TW: Tumor markers in gynecologic oncology. Obstet Gynecol Surv 45: 94-105, 1990.

35. Duk JM, de Bruijn HW, Groenier KH, Hollema H, ten Hoor KA, Krans M and Aalders JG: Cancer of the uterine cervix: Sensitivity and specificity of serum squamous cell carcinoma antigen determinations. Gynecol Oncol 39: 186-194, 1990.

36. Li J, Chen P, Mao CM, Tang XP and Zhu LR: Evaluation of diagnostic value of four tumor markers in bronchoalveolar lavage fluid of peripheral lung cancer. Asia Pac J Clin Oncol 10: 141-148, 2014.

37. Cao X, Zhang L, Feng GR, Yang J, Wang RY, Li J, Zheng XM and Han YJ: Preoperative Cyfra21-1 and SCC-Ag serum titers predict survival in patients with stage II esophageal squamous cell carcinoma. J Transl Med 10: 197, 2012.

38. Torre GC: SCC antigen in malignant and nonmalignant squamous lesions. Tumour Biol 19: 517-526, 1998.

39. Ryu HK, Baek JS, Kang WD and Kim SM: The prognostic value of squamous cell carcinoma antigen for predicting tumor recurrence in cervical squamous cell carcinoma patients. Obstet Gynecol Sci 58: 368-376, 2015.

40. Moody CA and Laimins LA: Human papillomavirus oncoproteins: Pathways to transformation. Nat Rev Cancer 10: 550-560, 2010.

41. Ferenczy A and Franco E: Persistent human papillomavirus infection and cervical neoplasia. Lancet Oncol 3: 11-16, 2002.

42. Schiffman M, Castle PE, Jeronimo J, Rodriguez AC and Wacholder S: Human papillomavirus and cervical cancer. Lancet 370: 890-907, 2007. 